NUB-3120/95-Th

May 1995

hepph/9506056

\title{
Can Hidden Gauginos Form Condensates?
}

\author{
Haim Goldberg \\ Department of Physics \\ Northeastern University \\ Boston, MA 02115
}

\begin{abstract}
Supersymmetry breaking may be linked to the formation of gaugino condensates in a hidden sector. In this work, the process of formation of the condensate is examined in a cosmological context, using an effective field theory of the gaugino bilinear which provides a reasonable interpolation between the high- and low-temperature phases. The implementation of anomaly requirements generates a large potential barrier between the zero-condensate configuration and that of the true (SUSY-breaking) vacuum. As a consequence, the transition to bubbles of true vacuum may be subject to an enormous exponential suppression. This leads to the same difficulties with inhomogeneity of the universe which occurred in the original inflationary scenarios.
\end{abstract}




\section{Introduction}

Softly-broken supersymmetry constitutes a reference point of much of present day discussion of elementary particle physics. Yet a well-defined dynamical mechanism driving the soft-breaking remains an elusive goal for theorists. An extensively studied possibility is that associated with the formation of condensates of one or more gaugino bilinears in the confining phase of hidden Yang-Mills sector [1]. A non-zero expectation value for a gaugino bilinear does not in itself necessarily lead to SUSY-breaking [2]: the mechanism hinges on the presence of an additional hidden sector field (e.g. the dilaton) in the kinetic function of the gauge field. In this case, the gaugino bilinear can indeed generate a non-zero F-term for the other field, and thus break SUSY. Of course, many problems remain, such as the absence of physically well-motivated models, and the mechanism for the cancellation of the cosmological constant. Nevertheless, this picture does provide at least a heuristic focus for discussion.

The usual approach, in such discussions of SUSY-breaking, is to begin with the condensate at (or near) its stable-vacuum value, and to integrate it out, as a constraint field, in favor of the light dynamical variables (dilaton, moduli) whose further evolution one may wish to study. In this work, I will focus on the process of formation of the condensate itself, using an effective field theory in the framework of the standard cosmology. It will be seen that the anomaly constraints on the effective theory, delineated very early in the discussion of this topic by Veneziano and Yankielowicz [2], will play the determining role in the problems encountered. The global SUSY discussion in Ref. [2] need only be amended by more recent developments concerning SUSY-breaking in order to arrive at the major result of interest: in the weak coupling domain of the dilaton, the formation of the condensate is exponentially suppressed by a potential barrier whose origin are the anomalies of the supersymmetric Yang-Mills theory corresponding to scale and $U_{X}(1)$ (axial) transformations. (The latter is an

$R$-type symmetry.) Completion of the phase transition suffers from the same lack of a 'graceful exit' which proved so problematic for the original inflationary paradigm. 


\section{The Effective Lagrangian}

The dynamical framework of the discussion which follows is that of a supersymmetric Yang-Mills theory in the confining phase, supplemented by the presence of the 'stringy' objects, the dilaton $S$ and the moduli $\mathcal{M}$. (The symbol $T$ will be reserved for temperature.) Following Refs. [2] and [3], I will consider an effective Lagrangian based on the superpotential (for $S U(N)$ )

$$
W=\frac{1}{32 \pi^{2}} U \log \left(U^{N} e^{8 \pi^{2} S}\right) \times \text { modular function },
$$

where $U$ is the chiral superfield whose first component (also called $U$ ) is the gaugino bilinear. The effective Lagrangian will depend on the Kahler form $K\left(U^{*}, U\right)$ for the $U$-field:

$$
\begin{aligned}
\mathcal{L}_{\text {eff }}= & \mathcal{L}_{\text {kin }}-V \\
\mathcal{L}_{\text {kin }}= & K_{U^{*} U} \partial^{\mu} U^{*} \partial_{\mu} U+\text { kinetic terms for } S, \mathcal{M}, \ldots \\
V= & (\text { function of } S, \mathcal{M}) \cdot K_{U^{*} U}^{-1} \log \left(U^{*} / \mu^{\prime 3}\right)^{N} \log \left(U / \mu^{\prime 3}\right)^{N} \\
& \quad \quad \text { terms of } O\left(U^{*} U\right) / M^{2} .
\end{aligned}
$$

In Eq. (2),

- $K_{U^{*} U}=\partial^{2} K / \partial U^{*} \partial U$

- $M=M_{\text {Planck }}$

- $\mu^{\prime} \simeq M e^{-8 \pi^{2} S / 3 N}$

- the second term in $V$ constitutes the sum of the additional $S$ and $\mathcal{M} F$-terms, as well as the $-3|W|^{2} / M^{2}$ term of the supergravity potential.

The effect of the second term in $V$ is crucial: it will (one hopes!) displace the potential minimum at $U=\mu^{\prime 3}$ below that at $U=0$, and, with a zero cosmological constant, the needed cancellation will require a non-zero $F$-term, consequently breaking 
supersymmetry. In this paper no proposals are made for the machinery to accomplish these purposes; a viable model is simply asumed to exist, and the consequences are examined.

In order to proceed, the properties of the Kahler potential need to be specified. In this paper, the initial (and primary) focus will be on the scale invariant Kahler proposed in 2]

$$
K\left(U^{*}, U\right)=a\left(U^{*} U\right)^{1 / 3}
$$

where $a$ is a positive constant. This will provide an intuitively reasonable interpolation between the high and low temperature phases. It will, however, present ambiguities with respect to Witten's index theorem [四. A 'softened' form of the Kahler,

$$
K\left(U^{*}, U\right) \sim U^{*} U / \mu^{4}
$$

will also be discussed in Section 5 as an illustrative alternative: it will be seen to generate a dynamics whose high temperature phase is difficult to interpret.

With the Kahler form (3) it is convenient to rescale [2, 3] to a field $Y$ with canonical dimensions via $U=(Y / \sqrt{a})^{3}$, to obtain a working version of $\mathcal{L}_{\text {eff }}$ :

$$
\begin{aligned}
& \mathcal{L}_{\text {eff }}= \partial^{\mu} Y^{*} \partial_{\mu} Y+\text { kinetic terms for } S, \mathcal{M}-V(Y, S, \mathcal{M}) \\
& V(Y, S, \mathcal{M})=f(S, \mathcal{M}) \cdot\left(Y^{*} Y\right)^{2} \log (Y / \mu)^{3 N} \log \left(Y^{*} / \mu\right)^{3 N} \\
& \text {-terms of } O\left(\left(Y^{*} Y\right)^{3} / M^{2}\right)
\end{aligned}
$$

where

$$
\mu=\sqrt{a} \mu^{\prime} \sim M e^{-8 \pi^{2} S / 3 N}
$$

In the absence of other fields, the minima at $Y e^{2 \pi i k / 3 N}=\mu$ do not break supersymmetry [2]. The remaining terms will displace this minimum downward by an amount

$$
\epsilon \sim \mu^{6} / M^{2} \sim m_{3 / 2}^{2} M^{2}
$$

representing the difference between vacuum energies of the broken and unbroken phases. Once this minimum has been attained, further evolution in the $S$ and $\mathcal{M}$ variables will drive $V$ to its global minimum. 
The potential is not invariant under the $U(1)$ rotation of $Y$, reflecting the $U_{X}(1)$ anomaly; however, it does display the $Z(N)$ symmetry for $|Y|=\mu$. For real $\mu$, the minimum of the potential lies very near $|Y|=\mu$, (displaced slightly by the last term in Eq. (可)), and in what follows I will consider the evolution of $Y$ along the real axis. A sketch of the potential along the real $Y$-axis, for fixed $S$ and $\mathcal{M}$, and with the SUSYbreaking gap $\epsilon$ greatly exaggerated, is shown as the solid line in Fig. 1. Central to the development of the paper is a discussion, which follows, of the zero of the potential at $Y=0$.

\section{The Configuration $Y=0$.}

In the global supersymmetric limit $(M=0)$ the potential shows a field configuration at $Y=0$ which is degenerate with the $N$ vacua at $|Y|=\mu$. What is the meaning of this configuration? It is clearly tied to the choice of Kahler, since the 'softened' choice (田) will cause $V(U=0) \neq 0$, with the same superpotential. The Witten index for $S U(N)$ is $N$ [4], which is the number of vacua at $|Y|=\mu$. It may be, then, that the

effective theory (with the Kahler (3)) should be modified in order to support a breaking of the degeneracy between $Y=0$ and $Y=\mu$. However, requiring that the discussion of the phase transition make use of conventional field-theoretic methods places several constraints on the dynamical framework:

- the model should contain attainable field configurations with both zero and nonzero condensate;

- the model should allow a study of the phase transition using canonical finite temperature corrections;

- the finite temperature corrections should localize the field configuration to zero condensate at high temperature.

The finite-temperature discussion in the next section will show these requirements to be met by the Lagrangian (5). It will also be pointed out, in a parallel fashion, why 
the use of the softened Kahler (4) fails to meet the criteria above. Since a finite temperature environment breaks supersymmetry, one should perhaps not worry about the seemingly extra zero-temperature vacuum at $Y=0$, as long as $T \neq 0$. This will be the philosophy followed in this work. The transition to $T \simeq 0$ will be carried out as a continuation from $T \neq 0$.

\section{Evolution of the Condensate}

A salient feature of the potential $V$ can be immediately noted: if the gaugino bilinear is near zero at early times, then the stable vacuum at $|Y|=\mu$ can be attained only

(a) by thermal excitation over the barrier;

(b) via quantum tunneling through the barrier;

(c) by a classical roll if the condition $Y=\mu(S, \mathcal{M})$ is obeyed at all points along the roll. (This eliminates the barrier: see Eq. (5).)

It is this transition from zero to non-zero condensate, in a cosmological context, which is the subject of this study. The formation of critical bubbles of true vacuum via either thermal excitation or quantum tunneling (cases $(a)$ and $(b)$ above) is controlled by actions calculated from either $O(3)$ - or $O(4)$-invariant solutions to the classical field equations; thus, the simultaneous behavior in the complete field space of $Y, S$ and the moduli is technically required. This is difficult to implement; instead, I will calculate the transition in the $Y$-variable for some fixed average value of $S$ (ignoring the moduli), and extract a posteriori the constraint on this average value for the validity of the result. The single exception to this, case $(c)$, will receive separate comment in Section 8. Finally, because the only two mass scales are $\mu$ and $M$, I will not retain numerical factors of $O(1)$ in what follows, since they will be irrelevant in exploring the parametric dependence of the results on $\mu$ and $M$. 


\section{Critical Bubble Formation: $T \neq 0$.}

In order to discuss the finite temperature situation, I now give a brief sketch of the thermal environment which will be assumed for the situation at hand. Standard finite temperature field theory concepts will be used since the strength of the interactions is sufficient to maintain thermal equilibrium.

At temperatures $T \gg \mu$, the perturbative description of the theory is appropriate, so that $V$ in Eq. (2) is not meaningful. When $T \sim \mu$, the effective theory becomes applicable, with the zero-temperature potential given in (5) supplemented by the oneloop contribution [5, 6] to the free energy

$$
\begin{aligned}
\Delta V_{T}(Y, T) & \sim T^{4} \int_{0}^{\infty} d x x^{2} \ln \left(1-e^{-\sqrt{x^{2}+m^{2}(Y) / T^{2}}}\right. \\
& + \text { similar term for fermions }+C .
\end{aligned}
$$

Here $m^{2}(Y)=\partial^{2} V / \partial Y^{2}$, and the real part of $\Delta V_{T}$ will be used when $m^{2}<0$. The constant $C$ will eventually be fixed so that $\Delta V_{T}(Y=0)=0$; thus, $C \sim+T^{4}$.

Details of $\Delta V_{T}$ will not be required in the present consideration. It will suffice to approximate it (its real part, at least) by a function which runs from

$$
\Delta V_{T}(Y, T) \sim-T^{4}+T^{2} Y^{2}+C \sim T^{2} Y^{2}
$$

near $Y=0$ (the free energy of massless quanta in the false vacuum + large $T$ correction) to

$$
\Delta V_{T}(Y, T) \sim 0+C \sim T^{4}
$$

near $Y=\mu$ (only massive glueballs or 'hidden hadrons' in the true vacuum). The free energy of other (non-hidden) fields which interact only gravitationally with the hidden gauge fields will play no role in this discussion. The dashed curve in Fig. 1 represents $V(Y, T)=V(Y)+\Delta V_{T}(Y, T)$, the sum of the zero temperature potential and the correction. With these minimal properties, two observations are important:

(1) For $T \sim \mu$, the confining properties of the second term on the r.h.s. of Eq. (9) are expected to force the condensate to begin its evolution near $Y=0$; 
(2) no phase transition can occur until $T^{4} \lesssim \epsilon$. This defines a critical temperature

$$
T_{c} \sim \epsilon^{1 / 4} \sim \mu^{3 / 2} / M^{1 / 2} \sim 10^{12} \mathrm{GeV}
$$

The dashed curve in Fig. 1 is appropriate to $T>T_{c}$.

Thus, it is seen that the effective theory with the Kahler (3) satisfies the constraints itemized in Section 3. If one instead attempts a finite temperature modification of the effective theory with the softened Kahler (4) (this time with $U$ as the field variable), it is easy to check that $(a)$ the potential is singular at $U=0$; $(b)$ the finite temperature correction is negligible everywhere in field space (the effective mass $m^{2}(U)=\partial^{2} V / \partial U^{2}>\mu>T$ everywhere). Thus, there is no mechanism to support as initial condition $U=0$ in this theory, and it fails to meet the criteria for interpolation between $U=0$ and $U=\mu^{3}$ delineated in Section 3 .

The calculation of the nucleation rate now proceeds along standard lines [7]: one evaluates the stationary O(3)-invariant (Euclidean) action (i.e., the bubble energy) for the appropriate boundary conditions, and divides it by $T$, in order to obtain the Boltzmann exponent. Because of the large barrier and the small energy gap between false and true vacua, thin-walled bubbles are optimal [8], so that, for $T<T_{c}$, the three-dimensional action (the difference between true and false vacuum energies in a bubble of radius $R$ ) becomes, as a function of $R$,

$$
E(T) \approx-R^{3} \epsilon+R^{2} S_{1}(T)
$$

where $\epsilon$ was given in Eq. (7), and $S_{1}(T)$ is the one-dimensional action integrated through the bubble wall [8],

$$
S_{1}(T)=\int_{Y_{+}}^{Y^{-}} d Y\left[2(V(Y, T)]^{1 / 2}\right.
$$

with $V(Y, T)=V(Y)+\Delta V_{T}$. (In practice, we may take $Y_{-}=0, Y_{+}=\mu$.) Varying $E(T)$ with respect to $R$ gives for the critical bubble

$$
\begin{aligned}
& R(T) \sim S_{1}(T) / \epsilon \\
& E(T) \sim S_{1}(T)^{3} / \epsilon^{2} .
\end{aligned}
$$


At the low temperature $T_{c}$ there is negligible error in omitting the contribution of $\Delta V_{T}$ in calculating the surface term $S_{1}(T)$. With the use of Eq. (5), one obtains

$$
S_{1}\left(T<T_{c}\right) \simeq S_{1}(0) \sim \mu^{3}
$$

From Eqs. (14),(15), the critical bubble energy for $T<T_{c}$ is

$$
E\left(T<T_{c}\right) \sim E(0) \sim \mu^{9} / \epsilon^{2} \sim \mu(M / \mu)^{4}
$$

The bubble radius at nucleation is

$$
R\left(T<T_{c}\right) \sim R(0) \sim \mu^{3} / \epsilon \sim M^{2} / \mu^{3}
$$

which is just the de Sitter horizon $H^{-1}$ corresponding to the vacuum energy $\epsilon$. Thus, general relativistic corrections are expected to be of $O(1)$ at most. (Further discussion will be given in the next section.) At this point, I also note that the thin-wall approximation is justified: the thickness of the bubble wall $\Delta R \sim \mu^{-1}$ [ 8 is much smaller than the the bubble radius $R \sim M^{2} / \mu^{3}$.

The Boltzmann exponent controlling thermal bubble nucleation for $T<T_{c}$ is

$$
B(T) \simeq E(0) / T \geq\left(M^{4} / \mu^{3}\right) / T_{c} \sim(M / \mu)^{4.5}
$$

which gives an average nucleation rate/unit volume of

$$
\Gamma / V \leq \mu^{4} e^{-B\left(T_{c}\right)}
$$

It is clear from Eq. (18) that the Boltzmann exponent is huge unless $\mu \gtrsim 0.1 M$. From Eq. (6) this proximity of $\mu$ and $M$ is possible only if $S$ is in a domain corresponding to strong gauge coupling at the time of the nucleation. A compelling argument that this is not the case has been made by Brustein and Steinhardt [9]: if the dilaton were indeed in the strong (gauge) coupling regime $S \lesssim 1$ at the time of gaugino condensation, its large potential energy in this configuration would carry it quickly to $S \rightarrow \infty$, beyond its stable vacuum value. If it is assumed that this is not the case, then the large result for $B$ indicates ipso facto that the $T \neq 0$ calculation represents an upper bound on the transition rate: for $T<T_{c}$ the energy density of the universe is dominated by the 
vacuum energy $\epsilon$, and the temperature will cool to zero in a few Hubble times of the de Sitter expansion, much smaller than the average time $t_{N}$ to nucleate a bubble:

$$
t_{N} \sim\left(\Gamma / V \cdot H^{-3}\right)^{-1} \sim H^{-1}(H / \mu)^{4} e^{B(T)} \sim H^{-1}(\mu / M)^{8} e^{B(T)} \gg H^{-1} .
$$

Thus, cooling to $T \simeq 0$ takes place well before any thermal nucleation out of the false vacuum. In order to circumvent the ambiguity (raised in Section 3) of reconciling the configuration $Y=0$ with the zero-temperature index theorem, I will keep the temperature small but non-zero $\left(T \ll T_{c}\right)$. This will allow a calculation of the quantum tunneling rate in a zero-temperature formalism.

\section{Quantum Tunneling: $T=0$.}

The calculational procedure here is well-known [8]: the decay of the false vacuum proceeds via the $\mathrm{O}(4)$ invariant Euclidean instanton solution connecting $Y \simeq 0$ and the appropriate point in $Y$ beyond the barrier. For the same reason given in the $\mathrm{O}(3)$ discussion of the last section, the thin-wall approximation 8 for bubble formation will be appropriate.

The zero temperature decay rate of the false vacuum is given by

$$
\Gamma / V \sim \mu^{4} e^{-B_{0}}
$$

where $B_{0}$ is the $\mathrm{O}(4)$ bounce. From [8], this is (in the thin-wall approximation)

$$
B_{0} \sim S_{1}(0)^{4} / \epsilon^{3} \sim(M / \mu)^{6}\left(\sim 10^{33} \text { for } \mu \simeq \text { its true vacuum value }\right)
$$

where the zero temperature surface energy $S_{1}(0)$ was estimated in Eq. (15) [10. The bubble size is the same as estimated in Eq. (17),

$$
R \sim M^{2} / \mu^{3} \sim H^{-1}
$$

the horizon size. Again, general relativistic corrections can be expected to be of $O(1)[11$. 


\section{Implications of Eqs. (21) and (22)}

Barring strong coupling (see discussion following Eq. (19)), the problem posed by the results (21) and (22) is clear: associated with excessive supercooling, there result clustering inhomogeneities of true vacuum [13] which are incompatible with the observed smoothness of the visible universe. In order to avoid these inhomogeneities, it is necessary that the probability $\eta$ to nucleate a true vacuum bubble in a Hubble space-time volume not be $\ll 1$. [13]. In the present case, unfortunately,

$$
\eta=\Gamma / V \cdot H^{-4} \sim(M / \mu)^{8} e^{-(M / \mu)^{6}} \sim 10^{-10^{30}}
$$

Thus, if none of the hypotheses which led to Eq. (22) is modified, there appears to be a cosmological problem associated with hidden gaugino condensation as the progenitor to SUSY-breaking. In the concluding section, I will review the input which led to the result, and comment on some possible modifications which need to be explored.

\section{Classical Roll}

This possibility was mentioned as a technical option in the introductory section. A glance at the potential $V(Y)$ in Eq. (5) shows that the barrier would be absent for a path in field space $Y=\mu(S)$. (I will omit the moduli from the discussion for purposes of simplicity.) Such a path obviously exists, but its probability will be exponentially suppressed unless it is a solution to the classical equations. This requirement imposes very special conditions on the dynamical variables (such as the Kahler potential for the dilaton); at present these seem somewhat ad hoc, but perhaps this option merits further study.

\section{Remarks and Summary}

(1) The principal source of difficulty for formation of the condensate is the presence of

$(\log Y)^{2}$ potential barrier in Eq. (5), which is a direct result of implementing the proper 
scale and axial anomalies in the effective theory. However, explicit implementation is not required in working with the elementary fields [14]. Of course, we have at present no method of calculating the evolution of the condensate through the phase transition using the elementary theory. In QCD with light quarks, there has been some evidence that an effective theory (i.e., the sigma model [15, 16]) can successfully model the chiral phase transition subsequently observed in lattice QCD [17.

(2) The results of this work carry over to the case of more than one condensate, since the condensates for product groups evolve independently.

(3) Matter fields have thus far not been included in the analysis, and I have not seen any simple way of characterizing their possible influence on the result. Their inclusion [18, 19] complicates the picture enormously, especially if they are massless [19, 20]. In the case of bilinear condensates $\Pi_{j}^{i}=Q^{i} \bar{Q}_{j}$ of fields in $N+\bar{N}$ representations of $S U(N)$, there is non-trivial kinetic mixing of the $\Pi_{j}^{i}$ with the gaugino bilinear $U$ [18] [21]. It is unclear whether this mixing could eventually destabilize the region of $U \simeq 0$ as a possible starting point for the evolution of the gaugino condensate. If it does not, then there would seem to be no immediate reason to modify the conclusions of this paper.

(4) To summarize, the principal result of this work is that the effective theory describing gaugino condensation also creates a barrier to a successful completion of the phase transition from zero to non-zero condensate. Only if the gauge theory is strongly coupled at the time of the transition (so that $\mu(S) \sim M$ ) can this difficulty be circumvented. But this would come at the price of unacceptable behavior for the dilaton after the phase transition. If gaugino condensation should become unfavored as a candidate to provide a superpotential for moduli, then perhaps matter condensates, in combination with stringy non-perturbative modifications manifested directly in the Kahler potentials of the moduli [22, 23], are responsible for SUSY-breaking.

I would like to thank Tom Taylor, Gabriele Veneziano, and Misha Voloshin for useful discussions during the course of this work, and for focussing attention on the questions surrounding the vacuum at $Y=0$ in the effective Lagrangian. This work was supported in part by Grant No. PHY-9411546 from the National Science Foundation. 


\section{Figure Caption}

Fig.1 Solid Curve: the zero temperature potential;

Dashed curve: the finite temperature potential for $T>T_{c}$, normalized to 0 at $Y=0$.

\section{References}

[1] S. Ferrara, L. Girardello, and H. P. Nilles, Phys. Lett. 125B 457 (1983); J. P. Derendinger, L. E. Ibàñez, and H. P. Nilles, Phys. Lett. 155B (1985) 65; M. Dine, R. Rohm, N. Seiberg, and E. Witten, Phys. Lett. 156B (1985) 55; C. Kounnas and M. Porrati, Phys. Lett. 191B (1987) 91; S. Ferrara, N. Magnoli, T. R. Taylor, and G. Veneziano, Phys. Lett. 245B (1990) 243; A. Font, L. Ibàñez, D. Lust, and F. Quevedo, Phys. Lett. 245B (1990) 401; H. P. Nilles and M. Olechowski, Phys. Lett. 248B (1990) 268; P. Binétruy and M. K. Gaillard, Phys. Lett. 253B (1991) 119; Nucl. Phys. B358 (1991)194; B. de Carlos, J. A. Casas, and C. Muñoz, Nucl. Phys. B399 623 (1993).

[2] G. Veneziano and S. Yankielowicz, Phys. Lett. 113B 231 (1982).

[3] S. Ferrara, N. Magnoli, T. R. Taylor, and G. Veneziano, Ref. [1]

[4] E. Witten, Nucl. Phys. B202 (1982) 253.

[5] L. Dolan and R. Jackiw, Phys. Rev. D9 (1974) 3320.

[6] S. Weinberg, Phys. Rev. D9 (1974) 3357.

[7] A. Linde, Phys. Lett. 70B (1977) 306; ibid 92B (1980) 119.

[8] S. Coleman, Phys. Rev. D15 (1977) 2929; C. Callan and S. Coleman, ibid. D16 (1977) 1762.

[9] R. Brustein and P. J. Steinhardt, Phys. Lett. 302B (1993) 196.

[10] The one-dimensional action (and the bounce) are the same in this case as would be expected for tunneling between local minima in a generic non-perturbative dilaton 
potential 223]: in that case, the potential barrier is lower $\left(\sim \mu^{6} / M^{2}\right.$ vs. $\mu^{4}$ in the present case); however, the width is larger ( $\sim M$ vs. $\mu$ in the present case).

[11] The effect on the bounce of including general relativity in the analysis was shown by Coleman and De Luccia [12] to constitute a multiplicative factor of $\left[1+\left(3 S_{1}^{2}\right) /\left(4 \epsilon M^{2}\right)\right]^{2} \sim O(1)$ in the present case. This is so because the bubble radius $R \sim H^{-1}$, as calculated without metric corrections; the Coleman-DeLuccia construction (which ensures that $R \leq H^{-1}$ ) then provides a modification which is parametrically of $O(1)$.

[12] S. Coleman and F. De Luccia, Phys. Rev. D21 (1980) 3305.

[13] A. H. Guth and E. J. Weinberg, Phys. Rev. D23 (1981) 876; Nucl. Phys. B212 (1983) 321; S. W. Hawking, I. G. Moss, and J. M. Stewart, Phys. Rev. D26 (1982) 2681.

[14] A. C. Davis, M. Dine and N. Seiberg, Phys. Lett. 125B (1983) 487.

[15] H. Goldberg, Phys. Lett. 131B (1983) 133.

[16] R. D. Pisarski and F. Wilczek, Phys. Rev. D29 (1984) 338.

[17] R. Gupta, G. Guralnik, G. Kilcup, A. Patel, and S. R. Sharpe, Phys. Rev. Lett. 57 (1986) 2621; J. B. Kogut, H. W. Wyld, F. Karsch, and D. K. Sinclair, Phys. Lett. 188B (1987) 353; M. Fukugita, S. Ohta, Y. Oyanagi, and A. Ukawa, Phys. Rev. Lett. 58 (1987) 2515.

[18] T. R. Taylor, G. Veneziano, and S. Yankielowicz, Nucl. Phys. B218 (1983) 493.

[19] I. Affleck, M. Dine, and N. Seiberg, Nucl. Phys. B241 (1984) 493; Nucl. Phys. B256 (1985) 557.

[20] D. Amati, K. Konishi, Y. Meurice, G.C. Rossi and G. Veneziano, Physics Reports 162 (1988) 169. 
[21] Recall that since the evolution of the condensate toward its non-zero value is being studied, one cannot follow the normal procedure of integrating out the heavy condensate field to leave an effective theory of light composite matter fields.

[22] T. Banks and M. Dine, Phys. Rev. D50 (1994) 7454.

[23] T. Banks, M. Berkooz, S. H. Shenker, G. Moore, and P. J. Steinhardt, hepth/9503114, RU-94-93. 


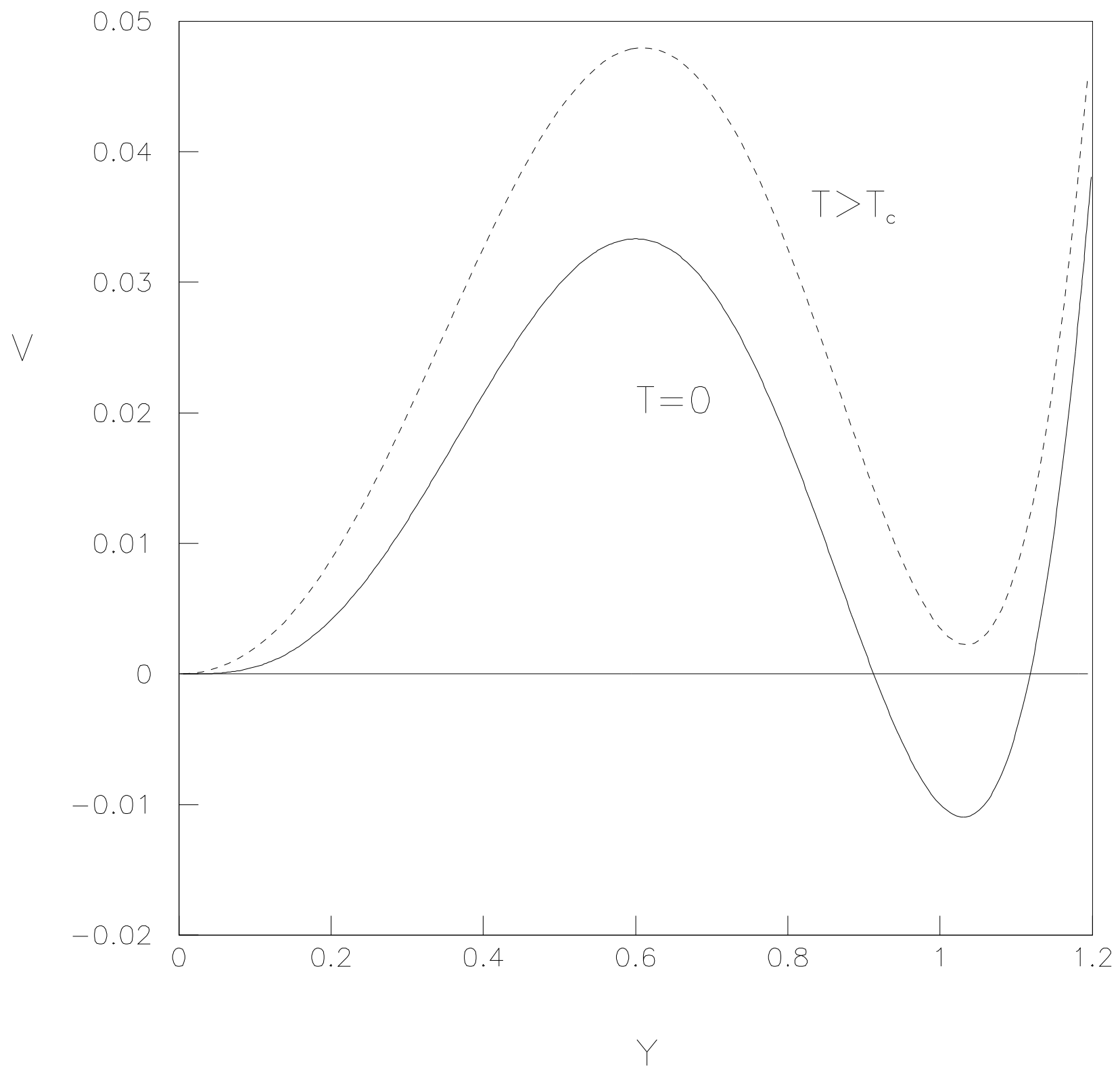

